\title{
Numerical Simulation of Static Noise Margin for a Six-Transistor Static Random Access Memory Cell with 32nm Fin-Typed Field Effect Transistors
}

\author{
Yiming $\mathrm{Li}^{1}$, Chih-Hong Hwang ${ }^{1}$, and Shao-Ming $\mathrm{Yu}^{2}$ \\ ${ }^{1}$ Department of Communication Engineering, National Chiao Tung University, \\ Hsinchu 300, Taiwan \\ ymli@faculty.nctu.edu.tw, chhwang@mail.ymlab.org \\ ${ }^{2}$ Department of Computer Science, National Chiao Tung University, Hsinchu 300, Taiwan \\ smyu@mail.ymlab.org
}

\begin{abstract}
We in this paper for the first time explore the static noise margin (SNM) of a six-transistor (6T) static random access memory (SRAM) cell with nanoscale silicon-on-insulator (SOI) fin-typed field effect transistors (FinFETs). The SNM is calculated with respect to the supply voltage, operating temperature, and cell ratio by performing a three-dimensional mixed-mode simulation. To include the quantum mechanical effect, the density-gradient equation is simultaneously solved in the coupled device and circuit equations. The standard deviation $\left(\sigma_{S N M}\right)$ of SNM versus device's channel length is computed, based upon the design of experiment and response surface methodology. Compared with the result of SNM for SRAM with 32nm planar metal-oxide-semiconductor field effect transistors, SRAM with SOI FinFETs quantitatively exhibits higher SNM and lower $\sigma_{S N M}$. Improvement of characteristics resulting from good channel controllability implies that SRAM cells fabricated with FinFETs continuously maintains cell stability in sub-32nm technology nodes.
\end{abstract}

Keywords: FinFET, SRAM, modeling and simulation, computational statistics.

\section{Introduction}

Silicon-based metal-oxide-semiconductor field effect transistors (MOSFETs) have been building blocks for SRAM cells [1]. For 45nm fabrication technologies, planar MOSFETs encounter significant challenges to device performance and circuit stability [2]3]. The SRAM with diverse device structures, such as the thin-buried-oxide SOI MOSFETs and SOI FinFETs have been of great interest [5,6,7,8] due to good suppression of shortchannel effects and high area's efficiency [9, 10, 11, 12, 13, 14]. Study of SNM for SRAM with the $32 \mathrm{~nm}$ planar MOSFETs and SOI FinFETs helps technological development.

In this paper, SNM of a 6T SRAM cell, shown in Fig. 11, is examined with a mixedmode simulation. The three-dimensional (3D) device simulation is performed to calculate device current-voltage $(I-V)$ characteristics by solving a set of density-gradient drift-diffusion equations [13, 14] in the solution of coupled device and circuit equations [15, 16, 17]. For SRAM with the 32nm planar MOSFETs and SOI FinFETs, shown in the insets of Fig $1 \mathrm{~b}$, SNM is explored and compared. SNM is the minimum DCvoltage disturbance necessary to upset the state of SRAM cell [2]. It is quantified by the 


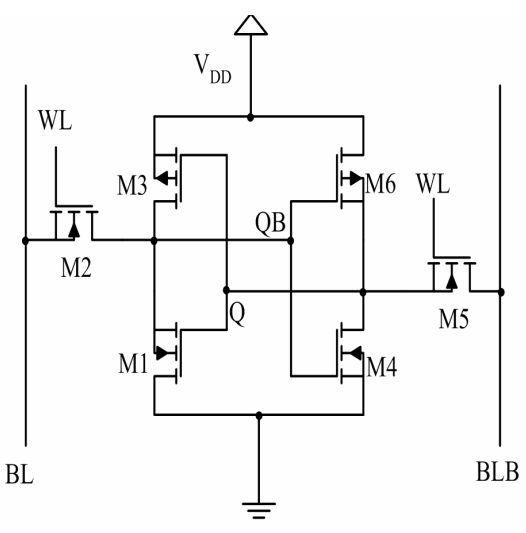

(a)

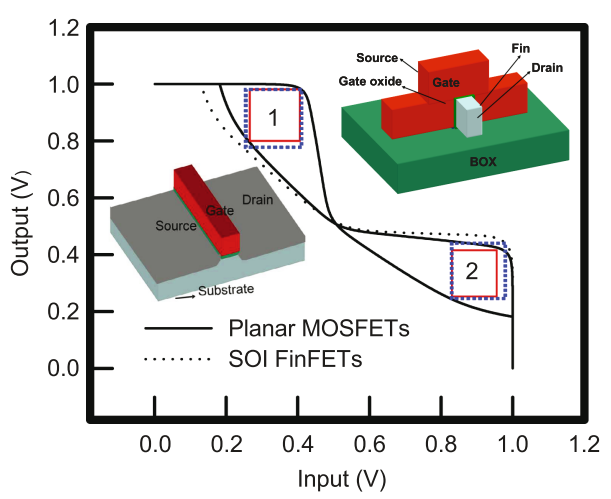

(b)

Fig. 1. (a) A circuit diagram of the simulated 6T SRAM, where the N- and P-typed devices are with the 32nm conventional planar MOSFETs and SOI FinFETs.(b) An illustration of the transfer characteristics of the SRAM with planar MOSFETs (the left inset plot) and SOI FinFETs (the right inset one). The edge of the maximum square is right the SNM. The blue squares are from SOI FinFETs and the red squares are from planar MOSFETs.

length of the side of the maximum square that can fit inside the butterfly curves formed by the cross-coupled inverters. The dependence of SNM, during the modes of hold and read, on the supply voltage, operating temperature, and cell ratio is examined. To explore the sensitivity of SNM, the standard deviation with respect to the device's channel length for the SRAM under the read mode is computed. The calculation is based on the computational statistics methodology [18], which integrates the design of experiment (DOE), a second-order response surface model (RSM), and the simulation of mixed-mode.

Simulation results imply that the SRAM with the $32 \mathrm{~nm}$ SOI FinFETs achieves dramatic improvements in the cell stability. For the mode of read, where the word line (WL) is biased at $V_{D D}$, the SNM of SRAM with SOI FinFETs has $20 \%$ increase, compared with the SRAM with planar MOSFETs. Improvement is evident when the cell ratio increases. The cell ratio means that the ratio of the widths of the pull-down transistor to the access transistor, shown in Fig. 11a. For the model of hold, the WL is equal to $0 \mathrm{~V}$, and a $6 \%$ improvement of SNM is observed for the SRAM with SOI FinFETs. Increase of the operating temperature leads to a decrease of SNM due to a reduction of the threshold voltage [1,2], but the SRAM with SOI FinFET exhibits that the SNM is less temperature dependence, compared with the SRAM using planar MOSFETs. Considering the Gaussian distribution to the variation of the device channel length, more than 2-times reduction of the standard deviation of SNM for the SRAM with SOI FinFETs is achieved, compared with the SRAM using planar MOSFETs.

This paper is organized as follows. In Sec. 2, we briefly state the simulation methodology. In Sec. 3, we report and discuss the simulation results. Finally, we draw the conclusions and suggest future work. 


\section{Simulation Methodology}

To assess the effect of device structure on the SNM of a 6T SRAM cell, a mixedmode simulation is directly adopted due to lack of industrial standard equivalent circuit model [19] for nanoscale SOI FinFETs. The coupled semiconductor device equations and circuit equations are iteratively solved for device electrical characteristics and SRAM's transfer characteristics. The results are used in the calculation of SNM for the SRAM with different devices. To consider the quantum mechanical effects in the mixed-mode simulation, 3D density-gradient is solved together with the drift-diffusion transport model for electrical characteristics of planar MOSFETs and SOI FinFETs [13, 14, 15, 17, 20]. The computed device characteristics are connected to the SRAM's circuit simulation. The formulation of SRAM's circuit equations is mainly based upon the Kirchhoff's current conservation law [21], shown in Fig. 1]. The ordinary differential equations are solved to estimate the nodal voltage and loop current, and then the DC transfer characteristics of SRAM are systematically computed with respect to different biasing condition, cell ratio, and operating temperature.

With the simulation of mixed-mode, the computational statistics methodology [18 22] is advanced to investigate the sensitivity of the SNM versus the device's channel length of the 6T SRAM cell. According to a face-centered-cube DOE, the simulation of mixed-mode is performed, and the simulation results are used in construction of the second-order RSM model of the SNM. The constructed RSM allows us to quickly analyze the sensitivity of SNM by assuming a proper distribution, such as a Gaussian distribution to the device's channel length.

\section{Results and Discussion}

For the $32 \mathrm{~nm}$ planar MOSFETs, we assume that the device width is equal to $50 \mathrm{~nm}$, the thickness of gate oxide is $1.5 \mathrm{~nm}$, the substrate doping concentration is $10^{18} \mathrm{~cm}^{-3}$ uniformly, and the source/drain doping is $10^{20} \mathrm{~cm}^{-3}$. To adjust device performance, compared with the $32 \mathrm{~nm}$ SOI FinFET, metal gate material with $4.7 \mathrm{eV}$ workfunction is selected in the $32 \mathrm{~nm}$ planar MOSFET. For the $32 \mathrm{~nm}$ SOI FinFETs, the fin height is $20 \mathrm{~nm}$, and the device width is $10 \mathrm{~nm}$. The source/drain doping is the same with the one of the planar device. To consider a lightly doping channel, the substrate doping concentration of SOI FinFET is $3 \times 10^{16} \mathrm{~cm}^{-3}$. To eliminate the short channel effects, we note that the setting on SOI FinFET's silicon fin thickness and the channel length has resulted in an optimal geometry aspect ratio (fin thickness/channel length) [6, 7, 9, 10, 11, 12, 13, 14]. A nearly undoped channel will reduce the effect of random dopant on the device performance [23]. Our 3D device simulation shows that SOI FinFETs not only have high ratio of the on- and off-state currents, but also possess a lower drain induced barrier height lowering (DIBL=39.88 $\mathrm{mV}$ ) and stable subthreshold swing ( $\mathrm{SS}=70.73 \mathrm{mV} / \mathrm{Dec}$ ), compared with the characteristics of planar MOSFET (DIBL $=95.19 \mathrm{mV}$ and $\mathrm{SS}=80.85 \mathrm{mV} / \mathrm{Dec}$ ).

By mirroring the inverter characteristics of transfer curves of input and output voltages, butterfly curves of the 6T SRAM operated under the read mode (the WL is biased at $V_{D D}$.), shown in Fig. $1 \mathrm{~b}$, are numerically computed. In this calculation, SRAM is with two different devices, where the $V_{D D}=1.0 \mathrm{~V}$, the operating temperature $=300 \mathrm{~K}$, and the 


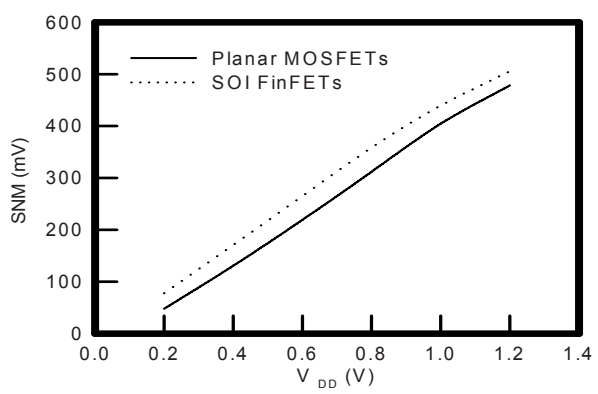

(a)

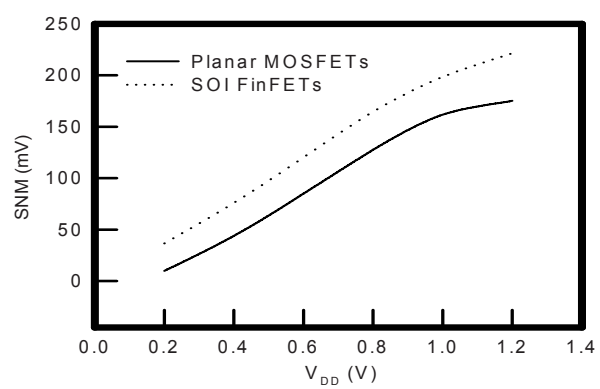

(b)

Fig. 2. SNM versus $V_{D D}$, under the (a) hold and (b) read modes, for SRAM with two device structures, where the cell ratio $=1$

cell ratio $=1$. We calculate the SNM with butterfly curves by finding an edge of a maximum square. The maximum square is given by the relation, $\operatorname{Min}\{$ square 1 , square 2$\}$, where the square 1 and the square 2 are the maximum squares locating in the upper and lower parts of the butterfly curves, respectively. The red and blue squares, shown in Fig. 1 $1 \mathrm{~b}$, are two maximum squares for the SRAM using different devices. The SRAM with SOI FinFETs achieves an improvement in SNM, compared with the result of SRAM with planar MOSFETs. Similarly, we have computed SNM for the hold mode, where the WL is $0 \mathrm{~V}$. To explore the characteristics of SRAM's SNM, variations of SNM with respect to different parameters are further examined. We note that increase of the operating temperature and reduction of $V_{D D}$ will induce a significant degradation of SNM in the SRAM cell. Figures $2 \mathrm{a}$ and $2 \mathrm{~b}$ show SNM varies with $V_{D D}$ for both the hold and read modes, where the cell ratio $=1$. Comparison between the results of $6 \mathrm{~T}$ SRAM with $32 \mathrm{~nm}$ planar MOSFETs and SOI FinFETs shows that SRAM with the latter building blocks possesses much better stability of SNM; in particular, for high supply voltage.

Under the hold mode, the SNM versus supply voltage for the SRAM with the planar MOSFETs and SOI FinFETs, is shown in Figs. 3 and 4 a, respectively. The SNM slightly is reduced when the cell ratio is increased. However, the change is insignificant due to an off-state operation of the transistors M2 and M5. The turned-off M2 and M5 keep the symmetry properties of the upper and lower squares in the butterfly curve and then maintain the original states at the nodes Q and QB. Therefore, an increase of the cell ratio does not significantly alter SNM for the SRAM under the hold mode. Under the read mode, for the SRAM with the planar MOSFETs and SOI FinFETs, SNM is depicted in Figs. $3 \mathrm{~b}$ and $4 \mathrm{~b}$, respectively. The SNM is increased when the cell ratio is increased under the read mode. It is a direct result due to high current resulting from the high cell ratio in the transistor M1, where the node Q is assumed at the logic "1" and the node QB is at the logic "0". The difference of SNM increases when the cell ratio is increased, for example, when the $V_{D D}=1.0 \mathrm{~V}$, the difference of SNM is about $100 \mathrm{mV}$ for the SRAM using SOI FinFETs and planar MOSFETs, respectively. The large difference comes from the nature of the device with triple-gate structure; in particular, for the driving transistors of SRAM cell. 


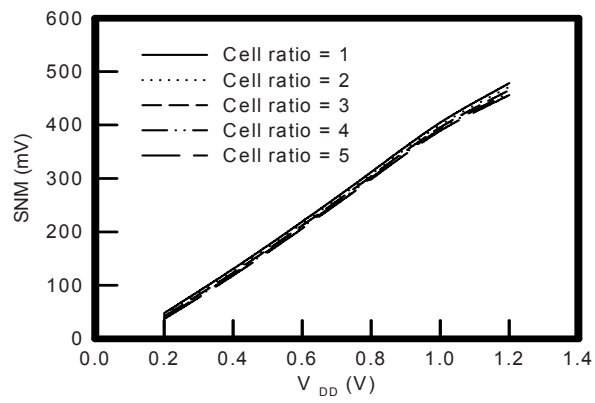

(a)

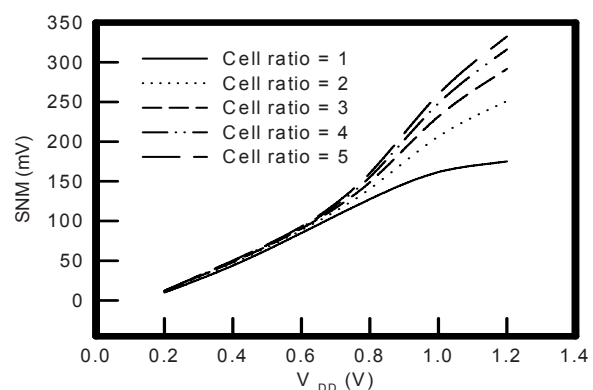

(b)

Fig. 3. The SNM versus $V_{D D}$ with respect to different cell ratios, under the (a) hold and (b) read modes, for the SRAM with 32nm conventional planar MOSFETs

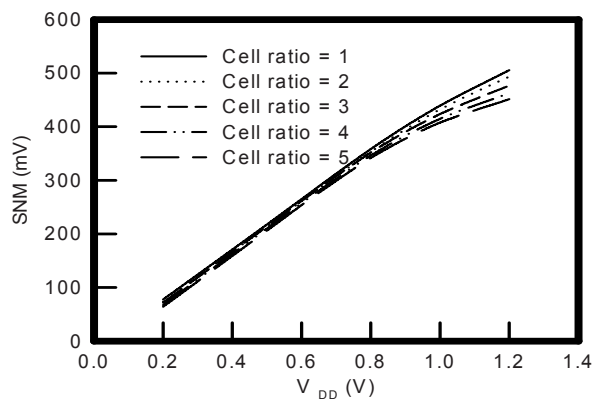

(a)

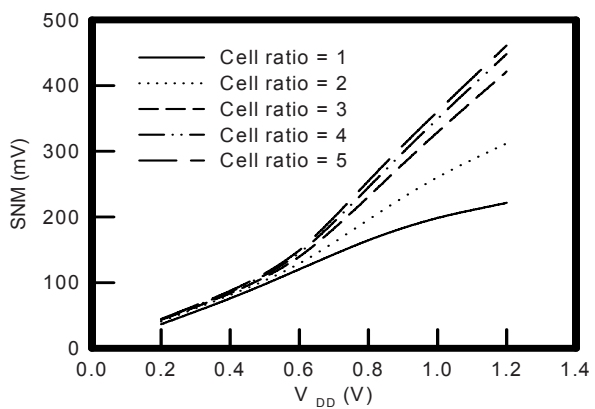

(b)

Fig. 4. The SNM versus $V_{D D}$ with respect to different cell ratios, under the (a) hold and (b) read modes, for the SRAM with $32 \mathrm{~nm}$ SOI FinFETs

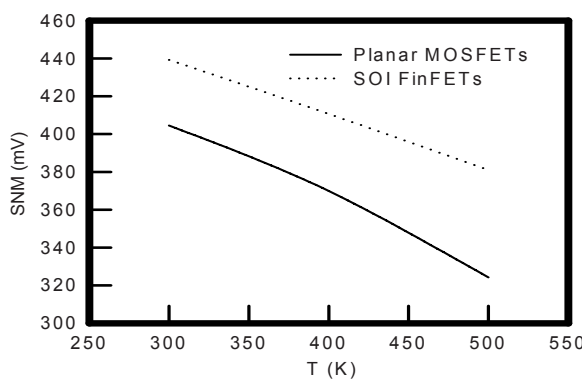

(a)

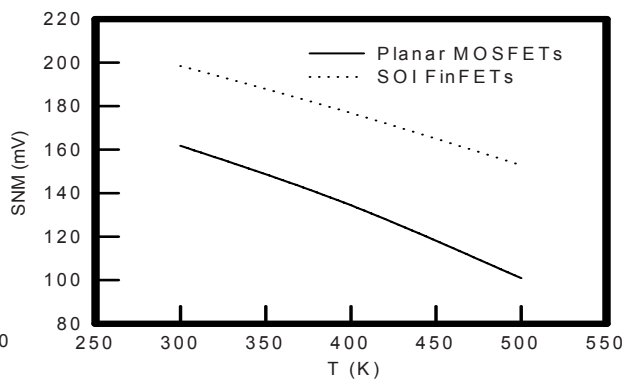

(b)

Fig. 5. The SNM versus the temperature for the SRAM with two device structures under the (a) hold and (b) read modes, where the supply voltage is equal to $1.0 \mathrm{~V}$ 
The SNM versus different operating temperature for the SRAM under the modes of hold and read are shown in Figs. 5a and 5b, respectively. The SNM, under the modes of hold and read, decreases when the temperature increases; it is attributed to decrease of the threshold voltage when the temperature increases. According to our calculation, the variation of threshold voltage versus temperature is almost the same between the planar MOSFETs and SOI FinFETs; nevertheless, the SNM of SRAM with 32nm SOI FinFETs is still less dependent upon the temperature (dotted-line in Fig. 5b) due to good channel controllability and improved short channel effects, compared with the SRAM using planar MOSFETs, in particular, for the SRAM under the read mode.

The sensitivity of SNM versus the channel length of transistors in the 6T SRAM cell is further investigated. We first model a second-order RSM for the SNM of 6T SRAM cell during the read mode, where the supply voltage is $1.0 \mathrm{~V}$ at the room temperature. Without loss of generality, we consider only the six devices' channel lengths. The RSM of SNM is in terms of the channel lengths of transistors M1, M4, and others including M2, M3, M5, and M6. We note the node Q is assumed to have a logic "0" and the node QB is with a logic"1", shown in Fig.11. The constructed RSM for the 6T SRAM using 32nm conventional planar MOSFETs and SOI FinFETs are given by:

$S N M=162.21+26.91 A-17.89 B+13.79 C-8.77 A^{2}-3.98 B^{2}+0.15 C^{2}-0.095 A C$,

and

$S N M=198.64+14.45 A-9.61 B+7.41 C-4.71 A^{2}-2.14 B^{2}+0.081 C^{2}-0.051 A C$,

respectively, where $A, B$, and $C$ are the channel lengths of M1, M4, and others, respectively. For the same model accuracy of the SNM of the 6T SRAM cell, the approach of RSM is computationally cost-effective, compared with an empirically fitted model. The Eqs. (1) and (2) suggest that the M1's channel length will result in a large impact on SNM. Therefore, we preliminarily explore the sensitivity of SNM versus the channel length of M1, shown in Fig. 6. Simultaneously assuming Gaussian distributions

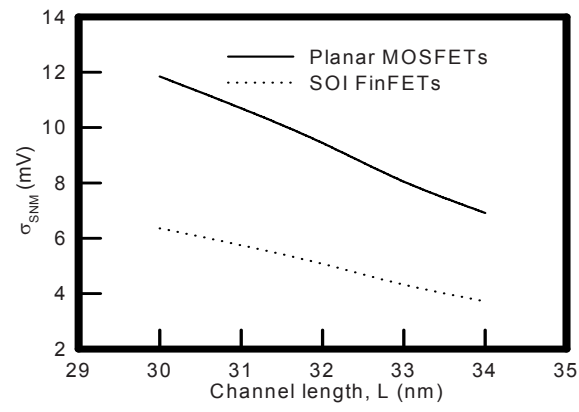

(a)

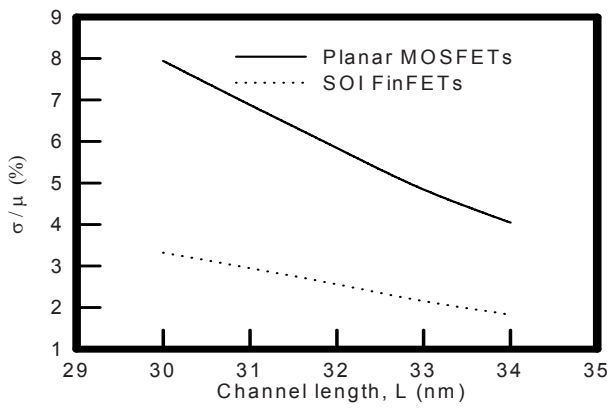

(b)

Fig. 6. (a) The standard deviation of SNM versus the M1's channel length for the SRAM using two device structures. (b) The normalized standard deviation of SNM versus the M1's channel length, where $\mu$ is the nominal value of SNM. 
on $A, B$, and $C$, where the 3 -sigma $=3 \mathrm{~nm}$ is assumed for each nominal value. The standard deviations of the SNM versus the channel length for the SRAM with $32 \mathrm{~nm}$ conventional planar MOSFETs (solid-line) and SOI FinFETs (dotted-line) are shown in Fig. 6a, where the cell ratio is $=1$. For the SRAM with conventional planar MOSFETs, $\sigma_{S N M}$ is larger than that of SOI FinFETs due to a serious short channel effects in planar MOSFETs. The plot of $\sigma_{S N M} / m u_{S N M}$ versus the M1's channel length is depicted in Fig. 6b. We notice that the difference of the ratio $(\sigma / \mu)$ becomes large if a uniform distribution for the device's channel length is assumed.

\section{Conclusions}

We have explored the SNM and its sensitivity for a 6T SRAM cell with the 32nm SOI FinFETs. Compared with the SRAM with conventional planar MOSFETs, the SRAM with SOI FinFETs possesses much better SNM and more stable variation of SNM under the modes of read and hold. The improvement of the cell stability attributes to good channel controllability and suppression of the short channel effects when the SRAM with SOI FinFETs. For more accurate estimation of the temperature effect on the SNM, the density-gradient equation could be solved with hydrodynamic model or Boltzmann transport equation instead of the drift-diffusion model. The SNM and its sensitivity can be subject to further investigation for the SRAM with other device structures, such as the bulk FinFET and nanowire FinFET. Impact of device parameters including line edge roughness and random doping on the fluctuation of SNM should also be considered in future works. We are currently designing device for the SRAM fabrication and the SNM comparison between theoretical estimation and experimental measurement.

\section{Acknowledgments}

This work was supported in part by Taiwan National Science Council (NSC) under Contract NSC-95-2221-E-009-336 and Contract NSC-95-2752-E-009-003-PAE, by MoE ATU Program, Taiwan, under a 2006-2007 grant, and by the Taiwan Semiconductor Manufacturing Company under a 2006-2007 grant.

\section{References}

1. Sze, S. M.: Physics of Semiconductor Devices. New York:Wiley. (1981)

2. Seevinck, E., List, F.J., and Lohstroh, J.: Static-noise margin analysis of MOS SRAM cells. IEEE J. Solid-State Circuits. 22(5) (1987) 748-754

3. Wakabayashi, H., Yamagami, S., Ikezawa, N., Ogura A., Narihiro, M., Arai, K., Ochiai, Y., Takeuchi, K., Yamamoto, T., and Mogami, T.:Sub-10-nm planar-bulk-CMOS devices using lateral junction control. Int. Electron Devices Meeting Tech. Dig. (2003) 20.7.1-20.7.3

4. Fischetti, M. V.: Scaling MOSFET's to the limit: A physicists's perspective. J. Comput. Electronics. 2(2-4) (2003) 73-79

5. Ananthan, H., Bansal, A., and Roy, K.: FinFET SRAM - device and circuit design considerations. Proc. 5th Int. Symp. Quality Electronic Design. (2004) 511-516 
6. Park, T., Cho, H.J., Choe, J.D., Han, S.Y., Jung, S.-M., Jeong, J.H., Nam, B.Y., Kwon, O.I., Han, J.N., Kang, H.S., Chae, M.C., Yeo, G.S., Lee, S.W., Lee, D.Y., Park, D., Kim, K., Yoon, E., and Lee, J.H.: Static noise margin of the full DG-CMOS SRAM cell using bulk FinFETs (Omega MOSFETs). Int. Electron Devices Meeting Tech. Dig. (2003) 2.2.1-2.2.4

7. Chen, H.-Y., Chang C.-Y., Huang, C.-C., Chung, T.-X., Liu, S.-D., Hwang, J.-R., Liu, Y.-H., Chou, Y.-J., Wu, H.-J., Shu, K.-C., Huang, C.-K., You, J.-W., Shin, J.-J., Chen, C.-K., Lin, C.-H., Hsu, J.-W., Perng, B.-C., Tsai, P.-Y., Chen, C.-C., Shieh, J.-H., Tao, H.-J., Chen, S.-C., Gau, T.-S., and Yang F.-L.: Novel 20nm hybrid SOI/bulk CMOS technology with $0.183 \mathrm{~m} 2$ 6T-SRAM cell by immersion lithography. VLSI Technology Symp. Tech. Dig.. (2005) 16-17

8. Samsudin, K., Cheng, B., Brown, A. R., Roy, S., and Asenov, A.: UTB SOI SRAM Cell Stability Under the Influence of Intrinsic Parameter Fluctuation. Proc. European Solid-State Device Research Conf. (2005) 553-556

9. Xiong, S. and Bokor, J.: Sensitivity of double-gate and FinFET Devices to process variations. IEEE Trans. Electron Devices. 50(11) (2003) 2255-2261

10. Park, J.-T. and Colinge, J.-P.: Multiple-gate SOI MOSFETs: device design guidelines. IEEE Trans. Electron Devices. 49(12) (2002) 2222-2229

11. Yang, F.-L., Chen, H.-Y., Chen, F.-C., Huang, C.-C., Chang, C.-Y., Chiu, H.-K., Lee, C.-C., Chen, C.-C., Huang, H.-T., Chen, C.-J., Tao, H.-J., Yeo, Y.-C., Liang, M.-S., and Hu, C.: 25 nm CMOS Omega FETs. J Int. Electron Devices Meeting Tech. Dig. (2002) 255-258

12. Li, Y. and Yu, S.-M.:Comparison of Threshold Voltage Fluctuations in Sub-45 nm Planar MOSFET and Thin-Buried-Oxide SOI Devices. Extended Abstracts Int. Solid State Devices and Materials Conf. (2006) 370-371

13. Li, Y., Chou, H.-M., and Lee, J.-W.: Investigation of Electrical Characteristics on Surrounding-Gate and Omega-Shaped-Gate Nanowire FinFETs. IEEE Trans. Nanotechnology. 4(5) (2005) 510-516

14. Li, Y.and Chou, H.-M.: AA Comparative Study of Electrical Characteristic on Sub-10 nm Double Gate MOSFETs. IEEE Trans. Nanotechnology. 4(5) (2005) 645-647

15. Palankovski, V., Belova, N., Grasser, T., Puchner, H., Aronowitz, S., and Selberherr, S.: A methodology for deep sub-0.25 gm CMOS technology prediction. IEEE Trans. Electron Devices. 48(10) (2001) 2331-2336

16. Grasser, T. and Selberherr, S.: Mixed-mode device simulation. Proc. 22nd Int. Conf. Microelectronics. 1 (2000) 35-42

17. Binder, T., Heitzinger, C., and Selberherr, S.: A study on global and local optimization techniques for TCAD analysis tasks. IEEE Trans. Computer-Aided Design of Integrated Circuits and Systems. 23(6) (2004) 814-822

18. Li, Y. and Chou, Y.-S.:A Novel Statistical Methodology for Sub-100 nm MOSFET Fabrication Optimization and Sensitivity Analysis. Extended Abstracts Int. Solid State Devices and Materials Conf. (2005) 622-623

19. Li, Y. and Cho, Y.-Y.: Intelligent BSIM4 Model Parameter Extraction for Sub-100 nm MOSFETs era. Jpn. J. Appl. Phys. 43(4B) (2004) 1717-1722

20. Li, Y., Sze, S. M., and Chao, T.-S.: A Practical Implementation of Parallel Dynamic Load Balancing for Adaptive Computing in VLSI Device Simulation. Engineering with Computers. 18(2) (2004) 124-137

21. Huang, K.-Y., Li, Y., and Lee, C.-P.: A Time Domain Approach to Simulation and Characterization of RF HBT Two-Tone Intermodulation Distortion. IEEE Trans. Microwave Theory and Techniques. 51(10) (2003) 2055-2062

22. Myers, R. H. and Montgomery, D. C.: Response surface methodology: process and product optimization using designed experiments. New York:Wiley. (2002)

23. Li, Y. and Yu, S.-M.: A Study of Threshold Voltage Fluctuations of Nanoscale Double Gate Metal-Oxide-Semiconductor Field Effect Transistors Using Quantum Correction Simulation. J. Comput. Electronics. 5 (2006) 125-129 